\title{
Linearly and circularly polarized laser photoinduced molecular order in azo dye doped polymer films
}

\author{
Bendaoud Saad* \\ National School of Applied Sciences of Safi, Cadi Ayyad University, Sidi Bouzid's Road, B P 63, Safi, 46000, Morocco
}

\begin{abstract}
Photo-induced behavior of Azo Disperse one (AZD1) doped Poly(Methyl MethAcrylate) (PMMA) using both linear and circular polarized light is studied. The anisotropy is not erased by the circular polarization light. The circular polarization light combined with relatively long lifetime of the cis state in azo dye doped polymers activate all transverse directions of the angular hole burning through the spot in the film inducing anisotropy. Under circular polarized light, there is no orientation perpendicularly to the helex described by the rotating electric field vector, trans molecules reorients in the propagation direction of the pump beam. The polarization state of the probe beam after propagation through the pumped spot depends strongly on the angle of incidence of both pump and probe beams on the input face. In the case where circular polarized pump and probe beams are under the same angle of incidence, the probe beam "sees" anisotropic film as if it is isotropic. Results of this work shows the possibility to reorient azobenzenetype molecules in two orthogonal directions using alternately linearly and circularly polarized beams.
\end{abstract}

\section{Introduction}

Amorphous azo dye doped polymers are very interesting for their potential applications in optics and photonics [1]. These potentialities come from the photoisomerization that modifies the molecular dielectric polarization and thus the refractive index as well as the absorption of the material. By using polarized light, one can induce collective molecular reorientation in a favored direction that leads to the anisotropy i.e., dichroism and birefringence. The optimization of these properties is important for example for All-Optical Switching [2] and Optical Data Storage [3], since modulations achieved are very high. If azobenzene molecules are excited using a linear polarized light, the angular hole burning occurs for the azo dye's transition dipole moment axis has a component parallel to the electric field (E-field) of the light [4]. An angular redistribution of trans molecules occurs when they relax from cis state to trans state. The molecules that are perpendicular to the E-field of the light are no longer pumped; the photo-isomerization process will tend to accumulate trans isomers in that direction that is the direction perpendicular to the oscillating E-field of the incoming light [5]. Circularly polarized light was used to erase the anisotropy in polymers containing azobenzene groups [6]. This technique of erase was reported for the first time by Rochon et al. [7]. Since then, while the theoretically use of this idea continue [8-14], no significant studies have been published for further verification.

In our previous study [15], a transient out of plane rotation of the director in azo dye doped nematic liquid crystals using linearly polarized light was observed. Although, the molecular reorientation in viscous substances such liquid crystals is completely different from that in solid matrices such PMMA; PS and PVA polymers. In viscous matrices, the reorientation can involve a collective motion of the polymer molecules hosts themselves whereas in solid matrices, the reorientation can involve only the azobenzene-type molecules while long polymeric chains not move. In azo dye doped solid polymer however, the photoisomerization of azobenzene-type molecules cause significant photo-mechanical deformations in the polymer matrix even at microscopic scale [16].Also, there is a big difference between azobenzene molecules bonded to (or in) the polymer chain and the unbonded one, hence the classification of these materials into two categories arises: azobenzene groups containing polymers (the copolymers) and azo dye doped polymers, and this is another subject that is outside our current talk.

Unpolarized light can push the azo molecules in viscous polymer containing azos [17] to orient in a direction which will be along the propagation direction of the excitation light. Dumont et al. are reported that using circular polarized light to pump Disperse Red one (DR1) doped in PMMA induces the angular redistribution and produces photo-assisted poling of the azobenzene molecules $[18,19]$. Barrett et al. are demonstrated that circular polarized light induces a reversible anisotropic dilatation due to the photomechanical effect which is due in turn to the photoisomerization in thin films of azo polymers [20]. Sajti et al. [21] are reported that upon illumination with circularly polarized light, the chromospheres of E1aP12 polymer tend to orient themselves towards the wave vector of the excitation light. Ramanujam et al. [22] are

Corresponding author: b.saad@uca.ma

(C) The Authors, published by EDP Sciences. This is an open access article distributed under the terms of the Creative Commons Attribution License 4.0 (http://creativecommons.org/licenses/by/4.0/). 
published a series of color pictures taken by a polarization microscope revealing that both linear and circular polarized light creates anisotropy in isotropic azobenzene polymers. Although, inducing the anisotropy using a linear polarized light is easy to understand owing to the reorientation of the azos molecules in directions perpendicular to the E-field direction of the excitation light. However, this fails to explain why circularly polarized light induces the anisotropy.

Nevertheless, if the light is circularly polarized, in principle, it's obvious that the E-field plane-of-vibration which rotates at the frequency of the light $\left(v \sim 5 \times 10^{14} \mathrm{~Hz}\right)$ will be prohibited from being occupied by the trans molecules [23]. Hence, it must be emphasized, however, that there is another favored direction during photoisomerization which will be along the propagation axis of a circular polarization of the incident excitation light. It is unavoidable that azobenzene-type molecules will efficiently buildup aligned along the propagation axis of the circular polarized light but this is often ignored in the literature, or interpreted as a photo-bleaching of the sample. Nunzi et al. are reported that circular polarized light not erase birefringence in cyano azobenzene polymer films, but induces the birefringence [24]; the authors have considered this as due to a phase transition in the studied liquid crystal polymer enhancing order. Chuang et al. [25] are published that the azobenzene molecules can be further aligned by recovering in films two incoherent pump beams of the light with crossed linear polarization states; this enhances the birefringence. In order to generalize the selective angular redistribution to the whole of a given sample, one possible way is trying to irradiate simultaneously several rectilinearly polarization directions. Since E-fields of nonpolarized light exists at each instant in all transverse directions, unpolarized light such power white light sources or unpolarized lasers can push the azo dyes to orient along the axis of the incoming light $[26,27]$, even sunshine is suitable $[28,29]$.

In our best acknowledgement, no work has been published on azo dye doped polymer films using circularly polarized pump and probe light irradiances. We report here the results of the photoinduced behavior of an azo dye doped polymer by using both linearly and circularly polarized light beams. Not only a circular polarized light not erase the photo-induced anisotropy but induces it in this azo dye doped polymer. Unambiguous results of the reorientation of azobenzene molecules towards the propagation direction of the light have been performed.

\section{Experimental descriptions}

\subsection{Fabrication of the films}

The azobenzene molecule used in this work is the azo dye called AZD1 [30-33]. The polymer is the poly(methyl methacrylate) abridged as PMMA (average Mw : $\sim 120,000$ by GPC powder, $\mathrm{Tg}=105^{\circ} \mathrm{C}$ ). Products are from Sigma-Aldrich. AZD1 doped in PMMA samples are obtained from the mixture of two separately prepared solutions. The first is the solution of the AZD1 in tetrahydrofuran (THF) solvent. The second one is the PMMA powder dissolved in the same solvent on a magnetic stirrer for 48 hours which was used to ensure the homogeneity of solutions and prevent the formation of eventual aggregates. The gravity deposition technique was used for deposing the solution on the microscope slides (Fisher Scientific Co.) previously cleaned and dried. Next, samples was left for $24 \mathrm{~h}$ in the closed, small Plexiglas box to get a regulated solvent evaporation rate allowing the mixture of azo dye and polymer solution to dry slowly on a leveled glass substrates under normal laboratory conditions $\left(20^{\circ} \mathrm{C}\right.$, $\mathrm{RH}=35-45 \%$ ) during one week in darkness. Films were then heated for $1 \mathrm{~h}$ at $100{ }^{\circ} \mathrm{C}$ in an oven to remove any remaining solvent [4]. The average thickness measured with a Sloan's Dektak IIA profilometer is $40 \mu \mathrm{m}$. Weakly doped films are analyzed with a Varian Cary $100 \mathrm{UV}-\mathrm{Vis}$ spectrophotometer, a typical spectrum is depicted in Fig. 1.

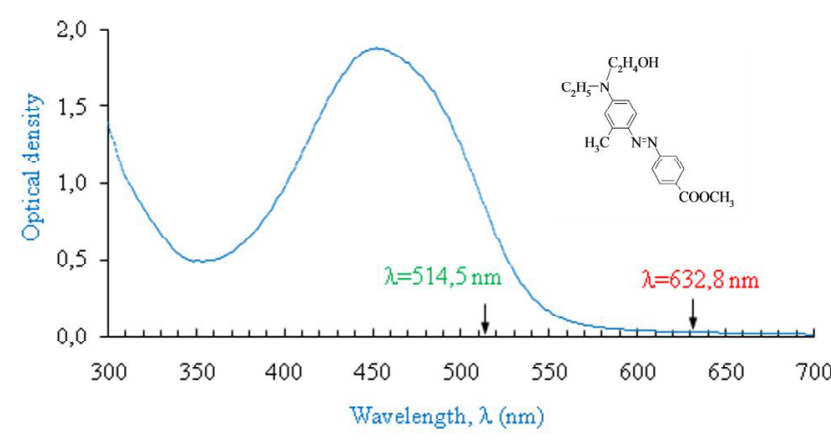

Fig. 1. UV-Vis absorption spectra of AZD1 doped PMMA film $(0,5 \mathrm{wt} \%)$. The inset shows the trans form of AZD1 molecular structure.

\subsection{Polarimetric experimental set-up}

In the experimental setup cited in many references, some examples are therein $[8, \mathbf{1 2}, \mathbf{2 4}, \mathbf{3 4 - 3 7 ]}$, pump and probe beams are usually linearly polarized and the polarization direction of the probe is oriented at an angle of $45^{\circ}$ with respect with that of the pump. Also, the film was set between a pair of crossed polarizer analyzer in the optical path of the probe beam. In general, the incidence angles of pump and probe beam are $0^{\circ}$ and $45^{\circ}$ respectively (or vice versa). However, this experimental setup is limited at least for three reasons.

First, the use of a rectilinear polarization beam restricts the angular hole burning to one direction; and only a certain percentage of azo molecules are activated. Second, only one transmittance component of the linearly polarized probe beam is detected after the analyzer. This leads to lose so much of data on what is happening in the sample. So change in the anisotropy was often interpreted as "randomization" and "erasure" $[2,35]$ if the transmittance of the probe beam decreases and as "photo-bleaching" $[8,38]$ if it increases whereas in reality, as our results will show, there is neither erasure nor photo-bleaching of the sample [21], photo- 
induced behaviors of polymers containing azos are simply a manifestation of reversible anisotropy changes in various forms introducing phase shift between vertical and horizontal components of the E-field of the probe beam leading to the change in its polarization state. The third reason is it does not allow us to probe the created anisotropy at different angles.

Our experimental set-up is shown in Fig. 2. The surface of the film is in Oxy plane. A continuous wave argon ion laser $\left(\lambda=514,5 \mathrm{~nm}, \phi_{\mathrm{e}} \sim 100 \mathrm{~mW}\right)$ was used as excitation source, and low power He-Ne laser $(\lambda=632,8$ $\mathrm{nm}, \phi_{\mathrm{e}} \sim 1 \mathrm{~mW}$ ) being a probe. The two wavelengths are placed on absorption spectrum (Fig. 1). The pump beam was fixed permanently at normal incidence on the input surface of photosensitive thin layer, i.e. parallel to $\mathrm{Oz}$ axis as shown in Fig. 2.

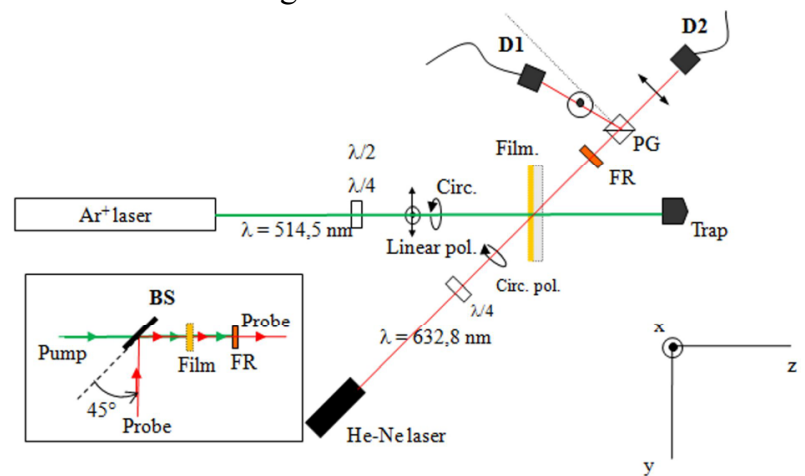

Fig. 2. Polarimetric Experimental setup.

The pump is a linearly polarized continuous wave argon ion laser beam emitting at $514,5 \mathrm{~nm}$. The probe beam is a linearly $\mathrm{He}-\mathrm{Ne}$ laser at $632,8 \mathrm{~nm}$. Optical components: $\lambda / 2$ and $\lambda / 4$ are half-wave and quarter-wave plates respectively. The PG component is the Glan-Taylor prism. Optoelectronic devices D1 and D2 are detectors of the light. The optical component FR is an interferential filter at $633 \mathrm{~nm}$. Inset the coaxially pomp and probe beam at incidence normal on the film.

The probe beam wave vector located in the Oyz incidence plane is for the moment under an angle of incidence of $45^{\circ}$ relative to normal incidence on the film. A linear polarized probe beam is circularly polarized by using a $\lambda / 4$-Plate at $\lambda \sim 632,8 \mathrm{~nm}$. The E-field of circular polarization state rotates at the frequency of the light $\left(\sim 4,74 \times 10^{14} \mathrm{~Hz}\right)$ scanning helically all-orthogonal directions to its wave vector especially in the film. A Glan-Taylor prism (Thorlabs Inc., Coating: 350 - 700 nm, extinction ratio: 100,000:1) was used to splits the circularly polarized probe beam into two equal rectilinear polarized components: the S-polarization component oscillates in the direction of the Ox axis and detected by the detector D1 and the P-polarization component in the incidence plane Oyz and detected by the detector D2. The P-component make an azimuth angle $\varphi$ with the Oy axis of $45^{\circ}$. The waists of the pump and probe beam at the plane of thin layer are approximately $2,94 \times 10^{-3} \mathrm{~m}$ and $0,52 \times 10^{-3} \mathrm{~m}$ respectively. Since the probe beam was centered in the illumined area of the film.

\section{Results and discussion}

\subsection{Induced anisotropy using P-polarization}

A fresh isotropic film (concentration 2,8wt\%, thicknesses $\sim 40 \mu \mathrm{m}$ ) were pumped using P-polarized beam for enough time to reach saturation then switched off. The irradiance is $3,61 \mathrm{~kW} / \mathrm{m}^{2}$ for the pump and $\sim 1,18 \times 10^{-3} \mathrm{~kW} / \mathrm{m}^{2}$ for the probe. To compare, the sunlight irradiance external to the Earth's atmosphere is $1,36 \mathrm{~kW} / \mathrm{m} 2$. Temporal behaviors of $\mathrm{S}-$ and Pcomponents of the probe beam are detected simultaneously. Figure 3 shows a complete cycle of photo-inducing anisotropy and its self-relaxation.

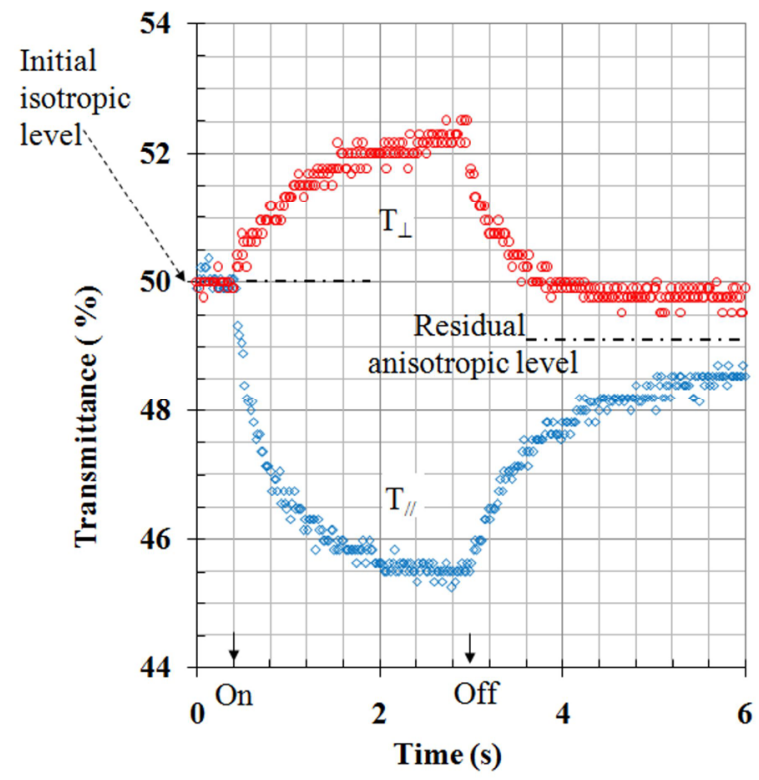

Fig. 3. One cycle of photo-buildup/self-randomization anisotropy in AZD1 doped in PMMA polymer film pumped by using CW Argon ion laser ( $\lambda=514,5 \mathrm{~nm})$.

Between the instants $\mathrm{t}=0$ and $\mathrm{t}=0,4 \mathrm{~s}$, fresh sample is not yet pumped. The $\mathrm{T}_{\perp}$ and $\mathrm{T}_{/ /}$transmittances corresponding respectively to $\mathrm{S}$ - and P-polarization components of the circular polarized probe beam are equal $\left(\mathrm{T}_{\perp}=\mathrm{T}_{/ /}=50 \%\right)$, indicating the random orientation of the azobenzene molecules [9]. In this case, the refractive indexes $n_{x}, n_{y}$ and $n_{z}$ corresponding to the $O x$, $\mathrm{Oy}, \mathrm{Oz}$ directions (see Fig. 2) are equal i.e., the effective refractive index ellipsoid is spherical. The S- and Pcomponents of the probe beam have in fact "seen" the same refractive index in propagating in their optical paths so the intensities of the S- and P-components are equal at each instant in this time interval.

Between the instants $t=0,4 \mathrm{~s}$ and $\mathrm{t}=3 \mathrm{~s}$, the pump light is turned on, the $T_{\perp}$ component of the probe beam increases while the $T_{/ /}$decrease, and the resulting anisotropy increases. The photo-isomerization continue until all the azo molecules which can participate in this mechanism lie in the perpendicular direction, then the anisotropy process is saturated [5]. In this time interval, a negative uniaxial medium was been photo-induced [25]. The optical axis of the medium is towards the Oy axis which is in the same direction of rectilinear Ppolarization of the pump beam, and its extraordinary and ordinary refractive indices are $\mathrm{n}_{\mathrm{e}}=\mathrm{n}_{\mathrm{y}}$ and $\mathrm{n}_{\mathrm{o}}=\mathrm{n}_{\mathrm{x}} \approx \mathrm{n}_{\mathrm{z}}$ 
respectively. Under pumping, S-polarization component of the circular polarized probe beam "see" the ordinary refractive index changes so the corresponding $T_{\perp}$ transmittance increases whereas the $\mathrm{T}_{/ /}$transmittance component corresponding to the P-polarization component decreases because it "see" a refractive index $\mathrm{n}(\varphi)$ changes given by the instantaneous equation of the index ellipsoid whereabouts $\mathrm{n}_{\mathrm{e}}(0)<\mathrm{n}(\varphi)<\mathrm{n}_{0}$. Indeed, an oblate ellipsoid of refractive indices whose extraordinary axis is along the Oy axis and the ordinary axis is along the Ox axis or Oz axis or any other axis in the Oxz plane passing through the origin $(0,0,0)$. The evaluated birefringence by using $\mathrm{I}=\mathrm{I}_{0} \sin ^{2}(\Delta \varphi / 2)$ where $\Delta \varphi=2 \pi \Delta \mathrm{nd} / \lambda[5,39]$ is $\Delta \mathrm{n}=\left(\mathrm{n}_{\mathrm{o}}-\mathrm{n}_{\mathrm{e}}\right) \sim 1,21 \times 10^{-3}$. The time required to achieve saturation takes only few seconds. Note that the transmittance (in absolute value) is higher for P-polarization component of the probe than for its Spolarization one.

At instant $t \sim 3$ s, pump light is turned off, the spontaneous relaxation starts $[18,19]$. There is no need to change the polarization state of the pump to randomize the oriented molecules, simply cut-off the light and the randomization starts itself i.e., auto-relaxation [30,31]. The whole inducing and self-randomization cycle is very fast (3-6 s). As one can see, the $T_{\perp}$ component of the probe beam decreases while the $\mathrm{T}_{/ /}$component increases "exponentially" and the resulting anisotropy decreases. This is due to the thermal reorientation of azobenzene molecules which tends to restore the thermodynamically randomized state $[7,40]$. Note that the final levels $\left(T_{\perp}\right.$ and $\mathrm{T}_{/ /}$) of both $\mathrm{S}$ - and P-components after switching off the pump tend towards another steady state level that is quite different from the initial level before the first pumping.

Thereafter, the film was cyclically pumped with the same P-linearly polarized beam. Figure 4 shows four inducing/relaxation cycles.

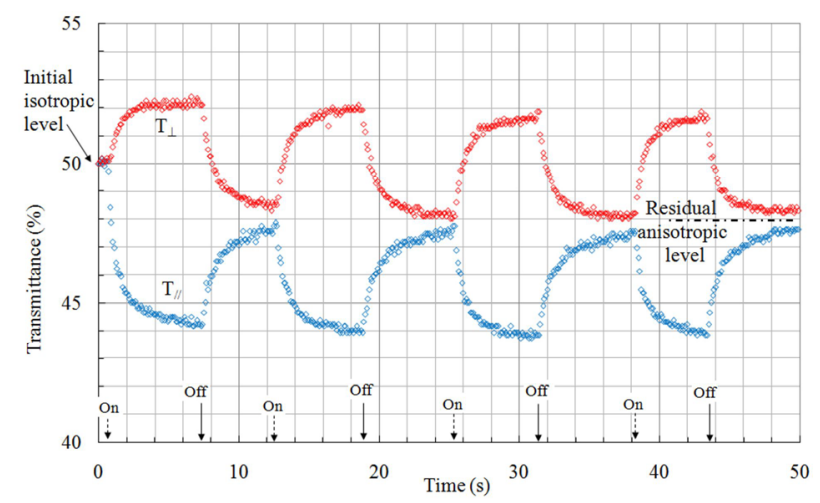

Fig. 4. Four photo-induced/relaxation cycles in AZD1 doped PMMA film cyclically pumped with On/Off P-polarized beam.

The first cycle in Fig. 4 is identical to that shown in Fig. 3. At instant $\mathrm{t}=13 \mathrm{~s}$ in Fig. 4, the P-polarized pump is turned on again on the same region (not in its amorphous state). As one can see, its effect is to produce the same amount of anisotropy in the same time interval like for the first cycle. During the second cycle and so on, the maximum birefringence reached at the saturation is $\Delta \mathrm{n} \sim 2,01 \times 10^{-3}$. A difference compared to the first cycle is that the $\mathrm{T}_{\perp}$ and $\mathrm{T}_{/ /}$transmittances are now symmetrical with respect to the horizontal line that indicates "persistent partial anisotropic level". This is because the pumped region is already partially ordered after the first irradiation and is not yet randomized completely. Indeed, at microscopic scale [38], build-up anisotropy again on the same irradiated region involves moving the azo dyes from a thermodynamically stable state with partially anisotropy to another oriented state identical to the previous one [40]. Even the initial isotropic level is never restored by pumping with the same beam whatever the direction of its linear polarization state, the reversible anisotropy, which was attributed to the molecular reorientation induced by the photo-isomerization of the azobenzene molecules, once no irreversible processes [8] that could indicate sample's photo-bleaching were observed.

\subsection{Alternately S- and P-polarizations}

A fresh film is pumped by using the same alternately Sand P-polarized beam. The results are shown in Fig. 5.

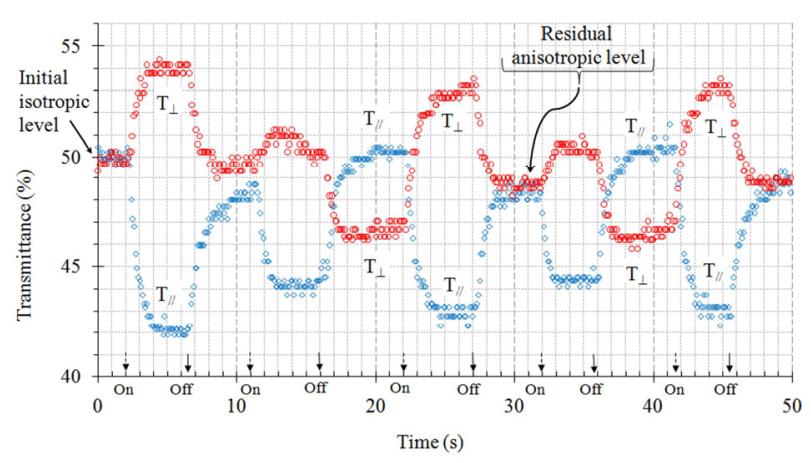

Fig. 5. Four photo-induced/relaxation cycles in a AZD1 doped PMMA film cyclically pumped with successively On/Off Sand P-polarized beam.

Consider for the moment the first cycle. Between instants $\mathrm{t}=0$ and $\mathrm{t}=2 \mathrm{~s}$, the sample is not yet pumped. At $\mathrm{t}=2 \mathrm{~s}$, the pump beam is switched on, the angular redistribution occurs subsequent to the photoisomerization process. Long axis of trans molecules will be gradually oriented perpendicularly to the E-Field of the pump beam i.e., in the Oy direction. The ordinary refractive index $n_{0}=n_{x} \approx n_{z}$ increases to a higher value, while the extraordinary refractive index $\mathrm{n}_{\mathrm{e}}=\mathrm{n}_{\mathrm{y}}$ decreases to the lower value i.e., $\mathrm{n}_{\mathrm{e}}=\mathrm{n}_{\mathrm{y}}<\mathrm{n}_{\mathrm{o}}=\mathrm{n}_{\mathrm{x}} \approx \mathrm{n}_{\mathrm{z}}$. Hence, the film does transform from an isotropic to a negative uniaxial medium [25]. One obtains an oblate ellipsoid of refractive indices with its extraordinary axis along the $\mathrm{Ox}$ axis and the ordinary axis is along Oy axis. At the saturation, the three principal refractive indices reach their dynamic equilibrium values, thus $n_{x}$ and $n_{z}$ are saturated to a larger value while $\mathrm{n}_{\mathrm{y}}$ to a smaller value in comparison with those of the isotropic film.

The second cycle in Fig. 5 show that exciting the film by using P-polarized beam immediately after the first irradiation with S-polarization state affect terribly the amplitude of $\mathrm{T}_{\perp}$ and $\mathrm{T}_{/ /}$transmittances. At $\mathrm{t} \sim 15,5 \mathrm{~s}$, the pump is switched off, $T_{\perp}$ decreases and $T_{/ /}$increases. 
Surprisingly they cross at $\mathrm{t}=17 \mathrm{~s}$ and $\mathrm{T}_{\perp}$ continue to decrease whereas $T_{/ /}$continue to increase towards completely different stable levels that are symmetrical with respect to final stable level reached at instant $t=29$ to $30 \mathrm{~s}$ or just before $\mathrm{t}=50 \mathrm{~s}$ (see Fig 5). After switched off the P-polarized beam, the amount of trans molecules that align in Ox direction continue to increase, and that's why $\mathrm{T}_{\perp}$ continue to decrease and $\mathrm{T}_{/ /}$to increase. In our best knowledge, the reorientation of the molecule in a proffered direction without light is new phenomenon has never been reported in the literature. This new behavior is probably due to a complex interactions between the azobenzene molecules and the matrix host subsequent to the photomechanical effect $[16,40,41]$.

The third cycle in Fig. 5 is obtained by pumping again using S-polarized. As one can see, the changes in $\mathrm{T}_{\perp}$ and $\mathrm{T}_{/ /}$probe components are reversible. The cycle starts at instant $\mathrm{t} \sim 22 \mathrm{~s}$. As the pump beam is switched on, azoic molecules reorient perpendicularly to the Spolarization plane. Therefore $\mathrm{T}_{\perp}$ component increases while $\mathrm{T}_{/ /}$decreases simultaneously, then they cross each other at point $\mathrm{t} \sim 22,5 \mathrm{~s}$ exactly at the residual stable partial anisotropy level. For the excited S-polarization state, the fifth cycle is identical to the third cycle and this latter is not identical to the first one, this because the start point is not the same. For the excited P-polarization, the fourth cycle is identical to the second cycle; and so on. More than hundred identical S- and P-polarized pump cycles have been achieved without the appearance of any signs of fatigue in the sample. It should be noted that the residual level at $\mathrm{t}=50 \mathrm{~s}$ (Fig. 4 and Fig. 5) is displaced downwards compared with the initial level of isotropy. At long-term; $\mathrm{T}_{\perp}$ and $\mathrm{T}_{/ /}$components tends towards another enduring steady state level which is quite different from the initial isotropic one; we have called it "partial residual anisotropic level" (Fig. 3). This behavior is a common characteristic of azo dye containing polymers films. Nevertheless, the thermal relaxation of molecules back to randomize trans state is incomplete: the anisotropy does not recover its initial isotropic value, either because of an incomplete cis-trans relaxation or because of some deformations of the doped polymer [42]. When the laser is turned off, a substantial number of cis azobenzene isomers remain "trapped" in their new orientation [7,40] and long-term macroscopic anisotropy has been optically.

\subsection{Linearly versus circularly polarizations}

Three principal polarimetric experimental configurations of interaction are studied. Fresh doped films has been used. Results are presented in Fig 6.

First, the pump beam is P-polarized. Curves labeled 1 correspond to this mode of excitation. Second, the pump is S-polarized. Curves labeled 2 correspond to this mode of excitation. Third, the pump beam is circularly polarized i.e., its E-field rotates helically around the $\mathrm{Oz}$ axis at the angular frequency of the light $\left(\sim 5 \times 10^{14} \mathrm{~Hz}\right)$. Curves labeled 3 correspond to this mode. Curves labeled 1(sp) and 1(pp) of the first cycle in Fig. 6 are the same as those shown in Fig. 3. For the instance, a curve 2(ps) corresponds to the P-polarization of the probe and S-polarization of the pump beams, and so on. The probing time interval for the first and second cycles are $\Delta \mathrm{t}=2,6 \mathrm{~s}$ and $\Delta \mathrm{t}=2 \mathrm{~s}$ respectively. Curves 2 and 3 consist each of an only one photo-induced/relaxation cycle. For these last, the pumping time interval is longer than this of curves 1. Saturation levels are almost accomplished. The most important finding is that the greatest anisotropy was induced when doped films were pumped by circularly polarized pump beam i.e., the circularly polarized light not erase anisotropy, but favors a certain order of the azoic molecules in azo dye doped polymer films.

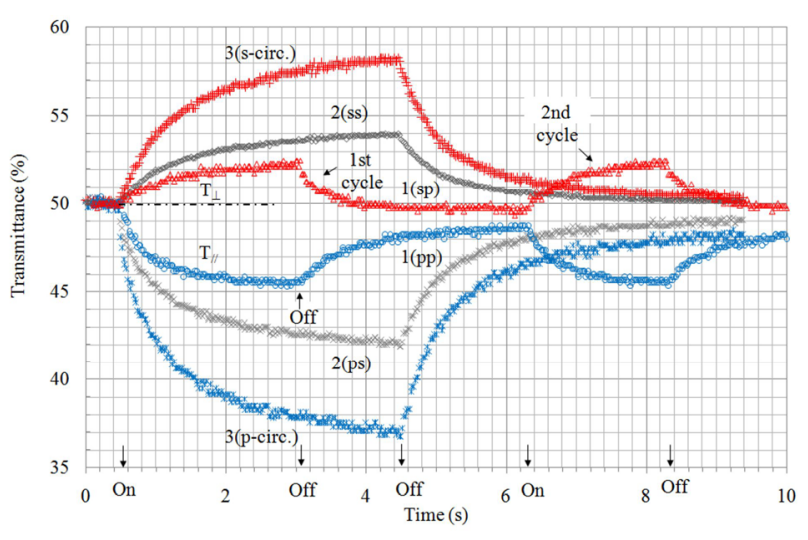

Fig. 6. Circularly compared to linearly polarized pump beam: the photo-induced anisotropy of AZD1 doped PMMA polymer dependence on the polarization state. The first letter after the curve number represents the probe beam's polarization state, and the second letter represents the excitation beam's polarization. Thus, for example, curve 1 corresponds to the vertically (s) rectilinear polarized probe and horizontally $(\mathrm{P})$ polarized excited beams with respect to the incidence plane. For each excited polarization, the excitation starts at $\mathrm{t}=0,4 \mathrm{~s}$.

On the other hand, it seems that the anisotropy induced by the S-polarized beam is greater than that induced by the P-polarized one. This is not true because the linearly polarized light selectively pump molecules parallel to its oscillating E-field, according to the model of the angular hole burning. Hence, the amount of the induced anisotropy should be the same for S- and Ppolarized light, the two cases are equivalent. This should be true whatever the rectilinear polarization direction in a given azo dye doped polymer. In all cases of rectilinear polarization state, the extraordinary axis of the created oblate ellipsoid of refractive indices will be oriented in the oscillating E-field direction of this polarization. Hence, the intensity difference that appears between 1 (sp) and 2(ss) curves, for example, is due to the orientation of the oblate index ellipsoid in the 3D space as "seeing" by the circular polarization of the probe beam combined to the effective thickness of the sample.

\subsection{Alternately linear and circular polarizations}

Fresh azo dye doped film was been pumped alternately by using successively the S-linear and circular polarization states of the same beam under the same experimental conditions. Results are shown in Fig. 7. 
Between $\mathrm{t}=0 \mathrm{~s}$ and $\mathrm{t}=1,5 \mathrm{~s}$, the sample is isotropic. The first cycle in Fig. 7 is assumed to be the same as the first one shown in Fig. 5. The second cycle was induced by using left-circular polarized beam, the third cycle by using again the S-polarized beam and the fourth cycle by right-circular polarization state of the pump. In general, switching off the pump beam leads to a considerable decrease of the resulting anisotropy. Moreover, no significant changes were observed by using left- or rightcircular polarized beam (see Fig. 7). Note that the induced anisotropy by using S-polarized beam during the third cycle (in Fig. 7) is slightly higher than that induced during the third and fifth cycles shown in Fig. 5. The third cycle in Fig. 7 was created after illuminating the film by the circular polarized beam while the third cycle in Fig. 5 was after the P-polarized one.

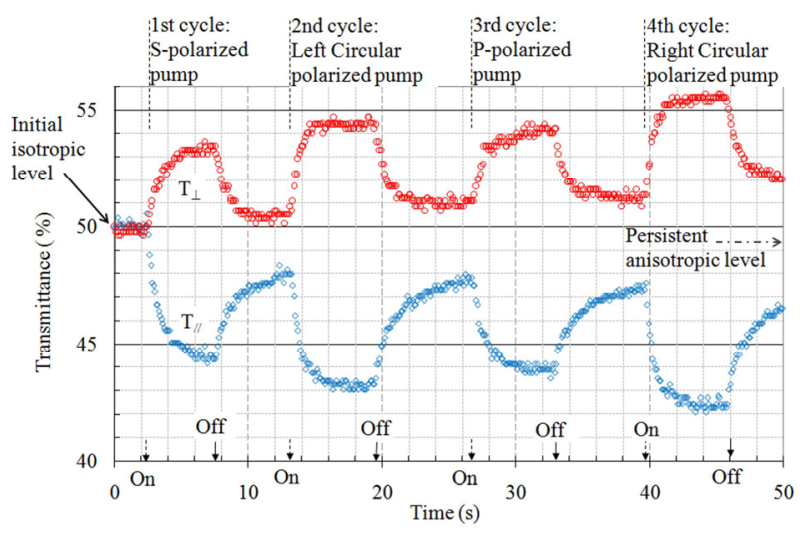

Fig. 7. Four photo-induced/relaxation cycles in a AZD1 doped PMMA film cyclically pumped with On/Off successively Slinearly and circularly polarized beam.

Also, another fresh doped film was irradiated alternately by using successively P-linear and circular polarization states of the same beam. The results are shown in Fig. 8. As one can see again, the circularly polarized pump light not erase residual anisotropy, but induce anisotropy which is important than that created by linearly polarized one.

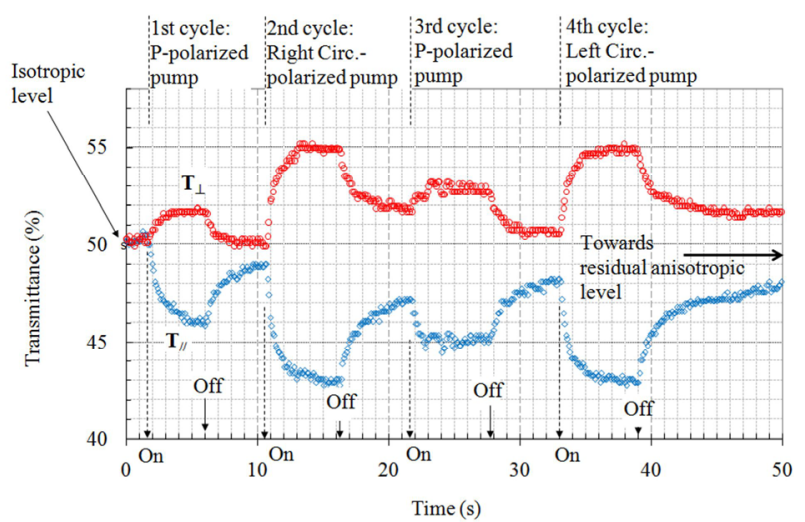

Fig. 8. Four photo-induced/relaxation cycles in a AZD1 doped PMMA film cyclically pumped with On/Off successively Plinearly and circularly polarized beam.

Generally when an azobenzene groups containing polymer film is irradiated with circular polarized light, the anisotropy is erased [8-14]. The use of this idea for almost polymers containing azobenzene molecules continues [1,43-45] and becomes widespread more and more. Our results however show that circular polarized light not only erase anisotropy but induces an anisotropy which is greater than that induced by linearly polarized one (see figures 7 and 8). Natansohn and Rochon [6] are pointed out that if the light is circularly polarized, there will be no preferred orientation within the film plane, but about the same amount of dichroic molecules will probably align in the homeotropic direction. The authors had added nothing to it, but the authors are reported one year before [38] that the progressively decrease of the circular dichroism as a function of number of photoinduced chirality/switching cycles in some azobenzene mesogens in their smectic phase is due to the tendency of the circular polarized light to orient the chromophores perpendicularly to its polarization, i.e., in the direction of propagation of the light. The idea however needed further investigations. Chun-I Chuang et al. [25] are used two different beams from two identical laser diodes at $532 \mathrm{~nm}$ to irradiate the Disperse Red 19 (DR19) doped in PMMA polymer. Their results show that the photoinduced anisotropy by superposing two beams with crossed linearly polarization states on a doped film is much higher than that obtained by using only a single beam. Those authors have stated that azo-dye molecules can be further aligned by the extra polarized pump light, thus increase the number of ordered molecules and enhance the induced birefringence. Nevertheless, our results show that during the irradiation with circularly polarized light, the trans molecules tends to orients in a direction perpendicular with the plane of the film which may induce some out-of-plane orientation, whereas inplane orientation would be minimal since the incoming beam is circularly polarized [20]. Otherwise, the circular polarization state was already used in order to selectively pump molecules parallel to the plane of the sample $[18,20]$ and to accumulate them in the perpendicular direction.

We have modified slightly the experimental setup shown in Fig. 2 to see is there some chirality induced or not. We have inserted a beam splitter $(\mathrm{R}=\mathrm{T}=50 \%)$ with high optical quality in the optical path of the pump beam between the $\lambda / 4$-plate and the film. This beam splitter (the component symbolized by BS inset Fig. 2) was mounted so that the pump and probe beams are at an incidence of $45^{\circ}$ (not the same angle) on the beam splitter. The optical power of the pump beam was adjusted again to $100 \mathrm{~mW}$. The results show that $\mathrm{T}_{\perp} \approx \mathrm{T}_{/} \approx 50 \%$. There is no significant change in the polarization state of the probe beam. Thus, no chirality was induced, according to the literature $[42,46]$. This observation reveals that the reorientation of the trans species occurs in such a way that the longitudinal molecular axis aligns in parallel with the propagation direction of the pump beam [21,47]. The film was transformed into a positive uniaxial medium whose optical axis is parallel to $\mathrm{Oz}$ axis; this is why the film seems as if it is isotropic if we probe it under the same 
angle of incidence as the pump beam. Anisotropy will not be ersed, only the optical axis will be changed.

Once the circularly polarized exciting light is turned on (see figures 6,7 and 8), the $\mathrm{T}_{\perp}$ component of the probe increases while its $T_{/ /}$decreases. The trans molecules align progressively towards the propagation direction of the pump beam i.e., in the Oz direction; so, in the case of circular polarized light, only the propagation direction is, in principle, perpendicular to the electric vector of the light which rotates around the propagation axis (the $\mathrm{Oz}$ axis in Fig. 2) at the angular frequency of the light. Therefore, the refractive index $\mathrm{n}_{\mathrm{x}} \approx \mathrm{n}_{\mathrm{y}}$ along the $\mathrm{Ox}$ and $\mathrm{Oy}$ axes respectively decreases to a lower value, while $n_{z}$ increases to the higher value by comparison to the index of the isotropic sample. The reorientational hole burning $[6,40]$ subsequent to the photo-isomerization push trans isomers to be accumulated parallel to the $\mathrm{Oz}$ direction, which is perpendicular to the pumping circular polarization state, as shown in Fig. 2. Thus, doped film does transform from an isotropic to a positive uniaxial medium whose optical axis is in the $\mathrm{Oz}$ direction. One obtains an oval index ellipsoid whose extraordinary axis is along the $\mathrm{Oz}$ axis and the ordinary axis is in Oxy plane $\left(\mathrm{n}_{\mathrm{o}}=\mathrm{n}_{\mathrm{x}} \approx \mathrm{n}_{\mathrm{y}}<\right.$ $\mathrm{n}_{\mathrm{e}}=\mathrm{n}_{\mathrm{z}}$ ). The experimental setup, shown in Fig. 2, is especially a powerful tool to study the angular redistribution behavior of azo dye doped polymers. Using circular polarized light allows us to pump and probe much better the samples. Taking into account the relatively long live time of cis state which is habitually in the order of few seconds in AZD1 doped PMMA polymer [30-33], the E-field of circular polarized light which rotates at the frequency of the light combined with the polarization state change after each coherence time [48] appears as if it activates the angular hole burning in all transverse directions in the film at each instant: it scans permanently the whole irradiated volume. Thus, relaxing molecules that undergoes the reverse reaction cis to trans will "forced" and not themselves to reorient in the propagation direction of the pump beam perpendicularly to the E-field of circularly polarized light, since only the propagation direction is perpendicular to the rotating E-field vector of the light. Under our experimental conditions, the action of circularly polarized light is to orient the trans molecules along its propagation direction.

Recently, Bin and Oates [49] have proposed a mathematical modeling based on light-induced anisotropic diffusion, in which the trans- (or cis) azomolecule is considered to be a microscopic dipole, determined by the microstructure (molecular conformation) of the trans (or cis) azo molecule. The results of this models show that in the case of a circularly polarized Gaussian laser beam, the spatial distribution of the trans vector align in the propagation direction of the laser beam. Of course, trans molecules aligned in this direction are usually "invisible" to a probe beam under normal incidence [6,37], as confirmed with our experimental setup (inset Fig. 2). This is also the case of the experimental setup in followings references $[35,50]$ and even many others.

\section{Conclusion}

We have studied the behavior of AZD1 doped PMMA polymer by using linearly and circularly pol arized laser beam and also circularly polarized probe He-Ne laser beam. Our results show that circular polarized light not erases anisotropy, but induces anisotropy which is much greater than that induced by linearly polarized light. The use of circular polarized light leading to activate all perpendicular directions of the angular hole burning in the illuminated sport in the polymer film intensifying the angular redistribution and enhances photo-induced anisotropy. Trans molecules align toward the propagation direction of the circular polarized light. The reorientation of azobenzene molecules successively in two orthogonal directions using cyclically linear and circular polarized beams of the light will be useful for applications such All-Optical switching and Optical Data Storage in particularly in the field of nanosceince and nanotechnology.

This work were supported by the - Fonds de Recherche du Québec-Nature et Technologies (FQRNT) - under the grant FT073270. The author thanks Marc-Antoine Blanchet for help.

\section{References}

[1] Y. ZHAO, T. IKEDA (EDS.). SMART LIGHT-RESPONSIVE MATERIALS. JOHN WILEY \& SONS, INC., NEW JERSEY, NJ. (2009)

[2] T. Xu, W. Zhu, Y. Lin, A. Liang. Optik 124, 305 (2013)

[3] T. D. Mislter. Opt. Phot. News. March, 28 (2005).

[4] T. Buffeteau, F. L. Labarthet, M. Pézolet, C. Sourisseau. Macromol. 31, 7312 (1998)

[5] T. Todorov, L. Nikolova, N. Tomova. Appl. Opt. 23, 4309 (1984)

[6] A. Natansohn, P. Rochon. Chem. Rev. 102, 4139 (2002)

[7] P. Rochon, J. Gosselin, A. Natansohn, S. Xie. Appl. Phys. Lett. 60, 4 (1992)

[8] C. R. Mendonça, U. M. Neves, L. De Boni, A. A. Andrade, D. S. dos Santos Jr., F. J. Pavinatto, S. C. Zilio, L. Misoguti, O. N. Oliveira Jr. Opt. Commun. 273, 435 (2007)

[9] C. R. Mendonça, L. Misoguti, A. A. Andrade, S. B. Yamaki, V. D. Dias, T. D. Z. Atvars, O. N. Oliveira Jr. Opt. Mater. 30, 216 (2007)

[10] A. Priimagi, S. Andriy. J. Polym. Sci., Part B: Polym. Phys. 52, 163 (2014)

[11] A. Goulet-Hanssens, T. C. Corkery, A. Priimagi, C. J. Barrett, J. Mater. Chem. C, 2, 7505 (2014)

[12] D. H. Choi. Bull. Korean Chem. Soc. 20, 1010 (1999)

[13] H. Z. Cao, W. Zhang, J. Zhu, X. R. Chen, Z. P. Cheng, J. H. Wu, X. L. Zhu. eXPRESS Polym. Lett. 2, 589 (2008)

[14] K. G. Yager, C. J. Barrett. J. Photochem. Photobiol. A: Chem. 182, 250 (2006) 
[15] B. Saad, T. V. Galstyan, M.-M. Denariez-Roberge, M. Dumont. Opt. Commun. 151, 235 (1998). Proc. SPIE, Int. Soc. Opt. Eng. 3418, 88 (1998). Opt. Lett. 23, 727 (1998)

[16] X. Ye, M. G. Kuzyk. Opt. Commun. 312, 210 (2014)

[17] Y. Wu, J.-I. Mamiya, A. Kanazawa, T. Shiono, T. Ikeda, Q. Zhang. Macromol. 32, 8829 (1999)

[198] Z. Sekkat, M. Dumont. Appl. Phys. B 54, 486 (1992)

[19] M. Dumont, A. El Osman. Chem. Phys. 245, 437 (1999)

[20] O. M. Tanchak, C. J. Barrett. Macromol. 38, 10566 (2005)

[21] S. Sajti, A. Kerekes, M. Barabás, E. Lőrincz, S. Hvilsted, P. S. Ramanujam. Opt. Commun. 194, 435 (2001)

[22] P. S. Ramanujam, O. B. Jensen, P. TidemandLichtenberg. Opt. Express 21, 1812 (2013)

[23] B. Saad. Int. J. Dev. Res. 6(11), 9946 (2016)

[24] E. Bagherzadeh-Khajeh Marjan, S. AhmadiKandjani, M. S. Zakerhamidi, J.-M. Nunzi. Opt. Mater. 38, 228 (2014)

[25] C.-I. Chuang, S.-H. Lin, Y.-F. Chao. Opt. Mater. 35, 366 (2013)

[26] K. G. Yager, C. J. Barrett. Chapter 8, 10-11: in H. S. Nalwa, (Eds.), Polymeric nanostructures and their applications. American Scientific Publisher. (2006) [27] M. Han, K. Ichimura. Macromol. 34, 82 (2001) [28] R. Yin, W. Xu, M. Kondo, C. C.Yen, J. I. Mamiya, T. Ikeda, Y. Yu. J. Mat. Chem. 19, 3141 (2009)

[29] K. Ichimura, M. Han. Chem. Lett. 3, 286 (2000)

[30] V. P. Pham, T. V. Galstyan, A. Granger, R. A. Lessard. Jpn. J. Appl. Phys. B 36, 429 (1997)

[31] V. P. Pham, G. Manivannan, R. A. Lessard, G. Bornengo, R. Po. Appl. Phys. A 60, 239 (1995)

[32] A. Yavrian, K. Asatryan, T. Galstian, M. Piché. Opt. Commun. 251, 286 (2005)

[33] A. Yavrian , T.V. Galstian, M. Piché, Opt. Mat. 26, $261(2004)$
[34] E. Mohajerani, N. H. Nataj. Opt. Mater. 29, 1408 (2007)

[35] Y. Luo, W. She, S. Wu, F. Zeng, S. Yao. Proc. of SPIE 5642, 298 (2005)

[36] S. Qi, C. Zhang, X. Yang, K. Chen, L. Zhang, X. Wang, T. Xu, J. Tian, G. Zhang. Optik 115, 253 (2004)

[37] F. F. Dall'Agnol, F. M. Shimizu, J. A. Giacometti. Chem. Phys. Lett. 608, 102 (2014)

[38] A. Natansohn, P. Rochon. Can. J. Chem. 79, 1093 (2001)

[39] F. Zhao, C. Wang, M. Qin, P. Zeng, P. Cai. Opt. Commun. 338, 461 (2015)

[40] A. Natansohn, P. Rochon, M. Pezolet, P. Audet, D. Brown, S. To. Macromol. 27, 2580 (1994)

[41] A. Natansohn, S. Xie, P. Rochon. Macromol. 25, 5531 (1992)

[42] P.-A. Blanche, Ph. C. Lemaire, M. Dumont, M. Fischer. Opt. Lett. 24, 1349 (1999)

[43] J. Vapaavuori. Doctoral Dissertation. Design of efficient photoresponsive azobenzene materials through supramolecular functionalization. Aalto University. (2013)

[44] A. Priimägi. Doctoral Dissertation. Polymerazobenzene complexes: from supramolecuar concepts to efficient photoresponsive polymers. Helsinki University of Technology. (2009)

[45] F. Filippo. Ph.D. Thesis. Photoinduced deformation in thin films containing azo-derivatives. Ecole

Polytechnique, ParisTech. (2009)

[46] Y. Kalachyova, O. Lyutakov, I. Goncharova, V. Svorcik. Opt. Mat. Express 5, 2761 (2015)

[47] A. Yavrian, T. V. Galstian, M. Piché. Opt. Eng. 41, 852 (2002)

[48] E. Hecht, Optics. $4^{\text {th }}$ ed., Addison Wesley, New York, NY. 2002

[49] J. Bin, W. S. Oates. Sci. Rep. 5, 1 (2015)

[50] T. Xu, G. Chen, C. Zhang, Z. Hao, X. Xu, J. Tian. Opt. Mat. 30, 1349 (2008) 